\title{
The Efficacy and Safety of Acarbose compared with Voglibose in Patients with Type 2 Diabetes: A Systematic Review and Meta-Analysis
}

Lian Liu', Song Wei Su², Yong $\mathrm{Xu}^{3}$, Qin Wan ${ }^{3}$, Xiao Ling Yang', Yu Ying Tang' and Hong Yan Sun ${ }^{1 *}$

${ }^{1}$ Nursing School, Southwest Medical University, Luzhou, P.R. China

${ }^{2}$ Basic Medical College, Guangzhou University of Chinese Medicine, Guangzhou University Island, P.R. China

${ }^{3}$ Department of Endocrinology, The Affiliated Hospital of Southwest Medical University, Luzhou, P.R. China

\begin{abstract}
Background: Acarbose and voglibose are alpha-glucosidase inhibitors and they are an effective therapy in patients with diabetes mellitus. Our aim is to directly compare the efficacy and safety of acarbose and voglibose for the treatment of patients with type 2 diabetes.

Methods: We searched the international web databases (PubMed, EMBASE, Cochrane Library, Web of Science) with an English language restriction (up to August, 2016). In addition, we checked bibliographies of each included study and the latest reviews to identify additional studies. For each clinical outcome, dichotomous data were analyzed by using the risk ratio $(\mathrm{RR})$ with the $95 \%$ confidence interval $(\mathrm{Cl})$. Continuous outcomes measured on the same scale and units were analyzed by using weighted mean differences (WMD) with the $95 \%$ confidence interval $(\mathrm{Cl})$; if continuous outcomes were measured on the different scale or units, it were analyzed by using standardised mean differences (SMD) with the $95 \%$ confidence interval $(\mathrm{Cl})$.

Results: There are no differences in glycemic control between acarbose and voglibose while adverse events in acarbose group were higher than voglibose group.

Conclusion: Our limited evidence finds that voglibose is more suitable for the treatment of type 2 diabetes compared with acarbose. Further well-designed and multicentric RCTs with larger sample are required to confirm these findings.
\end{abstract}

Keywords: Acarbose; Voglibose; Type 2 diabetes; Systematic review; Meta-analysis

\section{Introduction}

Type 2 diabetes, a gradual and complex disease with one characteristic of impaired insulin secretion, is difficult to treat availably in a long time $[1,2]$. Type 2 diabetes has become a global health problem because of a sharp rise in incidence [3]. The study have estimated that by 2030 , global adult ( 20 to 79 years) diabetes was estimated at 439 million and the prevalence was $7.7 \%$ [4]. The morbidity and mortality in type 2 diabetes will increase in the future, which causes serious burden for human beings.

Acarbose and voglibose are alpha-glucosidase inhibitors which restrain the increase in postprandial blood glucose levels through restraining as well as delaying digestion and absorption of carbohydrates; therefore they are an effective therapy in patients with diabetes mellitus [5-10]. Alpha-glucosidase inhibitors have been at the as first-line and second-line therapies in the treatment for patients with diabetes mellitus in Asian [11]. An increasing number of randomized controlled trials report the efficacy and safety of alphaglucosidase inhibitors in patients with type 2 diabetes, but their results are inconsistent. Thus, there are some systematic reviews [12-14] which assessed the efficacy and safety of alpha-glucosidase inhibitors in patients with type 2 diabetes.

Although the previous systematic reviews [12-14] had assessed the efficacy and safety of alpha-glucosidase inhibitors for patients with type 2 diabetes, there are still no any systematic reviews or meta-analysis that only directly summarize comparison data between acarbose and voglibose regarding the efficacy and safety for the treatment of patients with type 2 diabetes. We did a systematic review in order to directly compare the efficacy and safety of acarbose and voglibose for the treatment of patients with type 2 diabetes.

\section{Methods}

We reported this systematic review in accordance with preferred reporting items for systematic reviews and meta-analyses (PRISMA) [15].

\section{Data sources and searches}

The international web databases (PubMed, EMBASE, Cochrane Library, Web of Science) were searched with an English language restriction (up to August, 2016). We searched studies about the efficacy and safety of acarbose compared with voglibose in patients with type 2 diabetes from the international web databases using the following search terms: "diabetes mellitus, type 2", "niddm", "maturity-onset diabetes", "diabetes mellitus, noninsulin-dependent", "diabetes mellitus, adult-onset", "adult-onset diabetes mellitus", "diabetes mellitus, adult onset", "diabetes mellitus, ketosis-resistant", "diabetes mellitus, ketosis resistant", "ketosis-resistant diabetes mellitus", "diabetes mellitus, maturity-onset", "diabetes mellitus, maturity onset", "diabetes mellitus, non-insulin dependent", "diabetes mellitus, non-insulin-dependent", "non-insulin-dependent diabetes mellitus",

*Corresponding author: Hong Yan Sun, Nursing School, Southwest Medical University, No.3, 319 section of Zhong Shan Road, Jiangyan District, Luzhou 646000, P.R. China, Tel: 86-136-8820-6787; E-mail: sunhongyan234@163.com

Received December 24, 2017; Accepted December 31, 2017; Published January 01,2018

Citation: Liu L, Su SW, Xu Y, Wan Q, Yang XL, et al. (2018) The Efficacy and Safety of Acarbose compared with Voglibose in Patients with Type 2 Diabetes: A Systematic Review and Meta-Analysis. J Mol Biomark Diagn 9: 375. doi: 10.4172/2155-9929.1000375

Copyright: (c) 2018 Liu L, et al. This is an open-access article distributed under the terms of the Creative Commons Attribution License, which permits unrestricted use, distribution, and reproduction in any medium, provided the original author and source are credited. 
Citation: Liu L, Su SW, Xu Y, Wan Q, Yang XL, et al. (2018) The Efficacy and Safety of Acarbose compared with Voglibose in Patients with Type 2 Diabetes: A Systematic Review and Meta-Analysis. J Mol Biomark Diagn 9: 375. doi: 10.4172/2155-9929.1000375

Page 2 of 5

"diabetes mellitus, noninsulin dependent", "diabetes mellitus, slowonset", "diabetes mellitus, slow onset", "slow-onset diabetes mellitus", "diabetes mellitus, stable", "stable diabetes mellitus", "diabetes mellitus, type II", "maturity-onset diabetes mellitus", "maturity onset diabetes mellitus", "mody", "type 2 diabetes mellitus", "acarbose", "bay g 5421", "glumida", "lasa brand of acarbose", "precose", "glucor", "bayer brand of acarbose", "glucobay", "prandase", "voglibose", "basen", "random", and "randomized controlled trial". Furthermore, we performed a hand search, such as checking bibliographies of each included study and the latest reviews. All articles from the above search were transferred to Endnote X7 software so as to efficiently select qualified studies.

\section{Inclusion criteria and exclusion criteria}

We selected eligible studies according to the following criteria: (1) participants were patients with type 2 diabetes; (2) participants were randomly placed on voglibose (with or without other drugs) or acarbose (with or without other drugs); (4) publication language was English; (5) not less than one of outcomes (glycosylated hemoglobin (HbAlc), fasting blood glucose (FBG), postprandial blood glucose, triglycerides, total cholesterol, LDL (low-density lipoprotein), HDL (high-density lipoprotein) fasting insulin, postprandial serum insulin, fasting glucagon, postprandial serum glucagon, body weight, hypoglycemia, hyperglycemia, adverse event); (5) randomized controlled trials (RCTs) or randomised cross-over studies. Moreover, we also excluded the following studies: duplicate publication, studies without original data.

\section{Data extraction}

We made a spreadsheet using Microsoft Excel 2010 before extracting data. We extracted the following data from each included study: the first author; publication year; study design; sample size; characteristics of patient (age, sex); acarbose (dose, administration frequency, duration); voglibose(dose, administration frequency, duration); outcomes (glycosylated hemoglobin (HbAlc), fasting blood glucose (FBG), postprandial blood glucose, triglycerides, total cholesterol, LDL (low-density lipoprotein), HDL (high-density lipoprotein) fasting insulin, postprandial serum insulin, fasting glucagon, postprandial serum glucagon, body weight, hypoglycemia, hyperglycemia, adverse events). The above data was imported in the spreadsheet. The process of data extraction was independently performed by two authors. All inconsistent results in the process of data extraction were resolved by detailed discussion.

\section{Assessment of bias}

Two authors independently assessed the risk of bias for each eligible study using the Cochrane Collaboration's tool [16]. This tool includes seven items: random sequence generation; allocation concealment; blinding of participants and personnel; blinding of outcome assessment; incomplete outcome data; selective reporting; other bias. Each item has three options: low risk of bias; unclear risk of bias; high risk of bias. Authors selected each option according to detail description of each eligible study. All inconsistent results in the process of data extraction were resolved by detailed discussion.

\section{Statistical analysis}

For each clinical outcome, dichotomous data were analyzed by using the risk ratio (RR) with the $95 \%$ confidence interval (CI); if continuous outcomes were measured on the same scale and units, it were analyzed by using weighted mean differences (WMD) with the 95\% confidence interval (CI); if continuous outcomes were measured on the different scale or units, it were analyzed by using standardised mean differences (SMD) with the $95 \%$ confidence interval (CI). We selected a fixed or random model according to heterogeneity among all included studies. We evaluated heterogeneity among all included studies using I-square $\left(\mathrm{I}^{2}\right)$ [17]. If severe heterogeneity $\left(\mathrm{I}^{2}>50 \%\right.$ with an associated p-value $\leq 0.05$ ) occurred, the fixed model was chosen; otherwise, the random model was selected $[18,19]$. We used Stata 14.0 software performed these analyses. In addition, we used to funnel plots examed publication bias. We explored sources of heterogeneity using subgroup analysis, sensitivity analysis and meta-regression analysis.

\section{Results}

\section{Study selection and risk of bias summary}

We got a total of 104 articles from the international web databases and hand search. There were 70 articles after duplicates removed. We excluded 59 articles through reading titles and abstracts. Eleven articles were required to read full text. Finally, a total of 5 articles [11,20-23] were included in this systematic review. We summarized characteristics of each included study in Figure 1 and Table 1. Risk of bias summary was shown in Table 2.

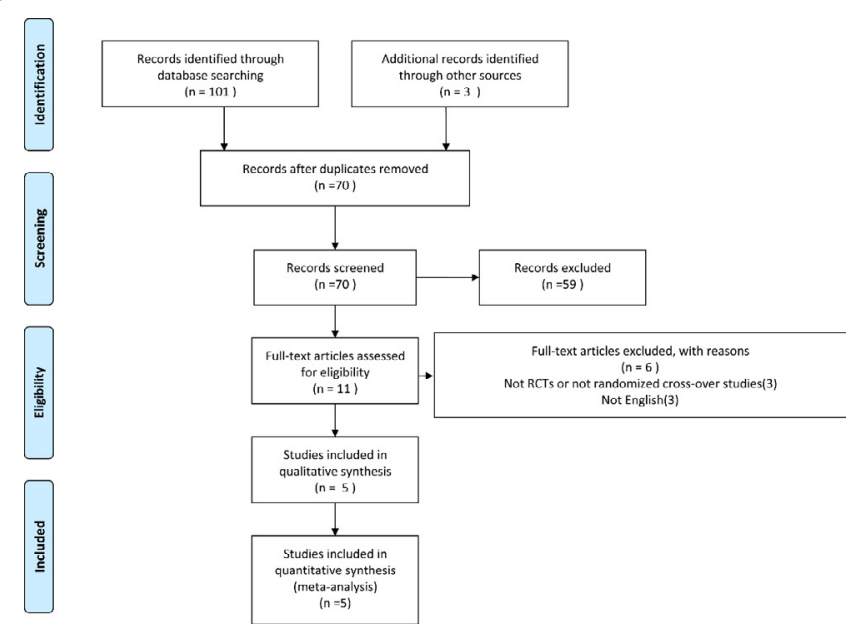

Figure 1: Flow diagram of study selection. RCTs: Randomized Controlled Trials.

\begin{tabular}{|c|c|c|c|c|c|}
\hline Study (Year) & $N(A / V)$ & $\begin{array}{l}\text { Mean } \\
\text { year }\end{array}$ & $\begin{array}{c}\text { Sex } \\
\text { (Male/ } \\
\text { Female) }\end{array}$ & Intervention & $\begin{array}{c}\text { Treatment } \\
\text { duration }\end{array}$ \\
\hline \multirow{4}{*}{ Ismail (2012) } & \multirow{4}{*}{$90(30 / 30)$} & \multirow{4}{*}{ NR } & \multirow{4}{*}{ NR } & $\begin{array}{c}\text { A: } 25 \mathrm{mg}(1 \text { month }) \\
\text { T.D.S }\end{array}$ & \multirow{4}{*}{4 months } \\
\hline & & & & $\begin{array}{c}50 \mathrm{mg}(3 \text { months }) \\
\text { T.D.S }\end{array}$ & \\
\hline & & & & $\begin{array}{c}\text { V: } 0.2 \mathrm{mg}(1 \text { month }) \\
\text { T.D.S }\end{array}$ & \\
\hline & & & & $\begin{array}{c}0.3 \mathrm{mg} \text { ( } 3 \text { months }) \\
\text { T.D.S }\end{array}$ & \\
\hline \multirow{2}{*}{$\begin{array}{l}\text { Young Lee } \\
(2014)\end{array}$} & \multirow{2}{*}{$\begin{array}{c}102 \\
(47 / 55)\end{array}$} & \multirow{2}{*}{$\begin{array}{c}\text { A: } \\
58.36 \\
\text { V: } \\
58.73\end{array}$} & \multirow{2}{*}{$\begin{array}{l}\text { A: } 29 / 30 \\
V: 33 / 22\end{array}$} & $\begin{array}{c}\text { A: Up to } 100 \mathrm{mg} \\
\text { T.D.S }\end{array}$ & \multirow{2}{*}{24 weeks } \\
\hline & & & & $\begin{array}{c}\text { V: Up to } 0.3 \mathrm{mg} \\
\text { T.D.S }\end{array}$ & \\
\hline \multirow{2}{*}{ Hitoshi (2014) } & \multirow{2}{*}{$81(22 / 19)$} & \multirow{2}{*}{$\begin{array}{l}\text { A: } 61.8 \\
\text { V: } 66.7\end{array}$} & \multirow{2}{*}{$\begin{array}{l}\text { A: } 13 / 9 \\
\text { V: } 8 / 11\end{array}$} & A: $300 \mathrm{mg} / \mathrm{day}$ & \multirow{2}{*}{12 weeks } \\
\hline & & & & $\mathrm{V}: 0.9 \mathrm{mg} / \mathrm{day}$ & \\
\hline \multirow{2}{*}{$\begin{array}{l}\text { Apichati } \\
(2002)\end{array}$} & \multirow{2}{*}{$30(30 / 30)$} & \multirow{2}{*}{ NR } & \multirow{2}{*}{ NR } & A: $100 \mathrm{mg}$ T.D.S & \multirow{2}{*}{8 weeks } \\
\hline & & & & V: 0.2 mg T.D.S & \\
\hline \multirow{2}{*}{$\begin{array}{c}\text { Watanabe } \\
(2004)\end{array}$} & \multirow{2}{*}{$20(10 / 10)$} & \multirow{2}{*}{$\begin{array}{l}\text { A: } 56.2 \\
\text { V: } 54.2\end{array}$} & \multirow{2}{*}{$\begin{array}{l}\text { A: } 5 / 5 \\
\text { V: } 4 / 6\end{array}$} & A: 300 mg T.D.S & \multirow{2}{*}{4 weeks } \\
\hline & & & & V: $0.9 \mathrm{mg}$ T.D.S & \\
\hline
\end{tabular}

Notes: A: Acarbose; V: Voglibose; NR: Not Report; T.D.S: Three Times One Day

Table 1: Characteristics of each included study. 
Citation: Liu L, Su SW, Xu Y, Wan Q, Yang XL, et al. (2018) The Efficacy and Safety of Acarbose compared with Voglibose in Patients with Type 2 Diabetes: A Systematic Review and Meta-Analysis. J Mol Biomark Diagn 9: 375. doi: 10.4172/2155-9929.1000375

Page 3 of 5

\begin{tabular}{|c|c|c|c|c|c|c|c|}
\hline $\begin{array}{l}\text { Study } \\
\text { (year) }\end{array}$ & $\begin{array}{l}\text { Random } \\
\text { sequence } \\
\text { generation }\end{array}$ & $\begin{array}{l}\text { Allocation } \\
\text { concealment }\end{array}$ & $\begin{array}{l}\text { Blinding of participants and } \\
\text { personnel }\end{array}$ & $\begin{array}{l}\text { Blinding of } \\
\text { outcome } \\
\text { assessment }\end{array}$ & $\begin{array}{l}\text { Incomplete } \\
\text { outcome data }\end{array}$ & $\begin{array}{l}\text { Selective } \\
\text { reporting }\end{array}$ & Other bias \\
\hline Ismail (2012) & Low & Unclear & High & High & Low & Low & Unclear \\
\hline $\begin{array}{l}\text { Young Lee } \\
(2014)\end{array}$ & Unclear & Unclear & Unclear & Unclear & High & Low & Low \\
\hline Hitoshi (2014) & Low & Unclear & Unclear & Unclear & Unclear & Low & Low \\
\hline Apichati (2002) & Unclear & Unclear & Unclear & Unclear & Low & Low & Unclear \\
\hline $\begin{array}{c}\text { Watanabe } \\
(2004)\end{array}$ & Unclear & Unclear & Unclear & Unclear & Low & Low & Unclear \\
\hline
\end{tabular}

Table 2: Risk of bias summary.

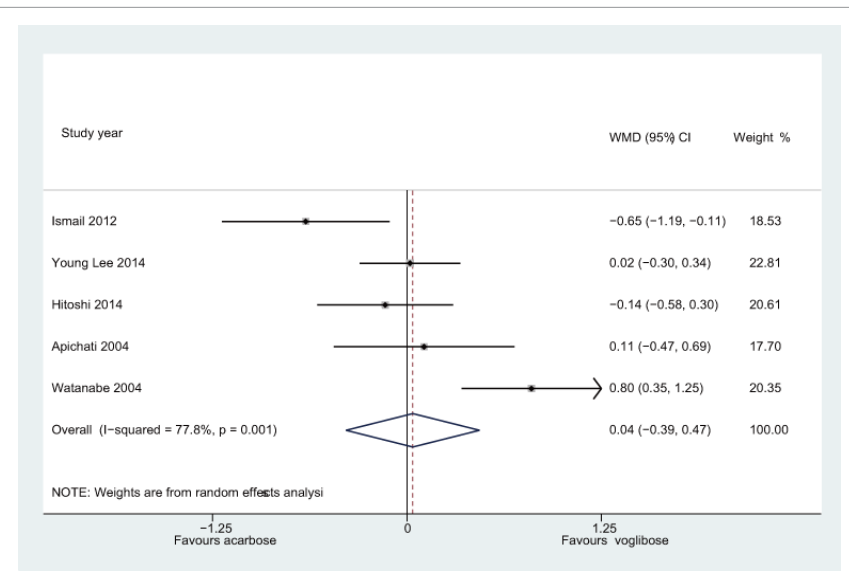

Figure 2: Weighted mean differences with $95 \%$ confidence interval in $\mathrm{HbA} 1 \mathrm{c}$ between acarbose group and voglibose group.

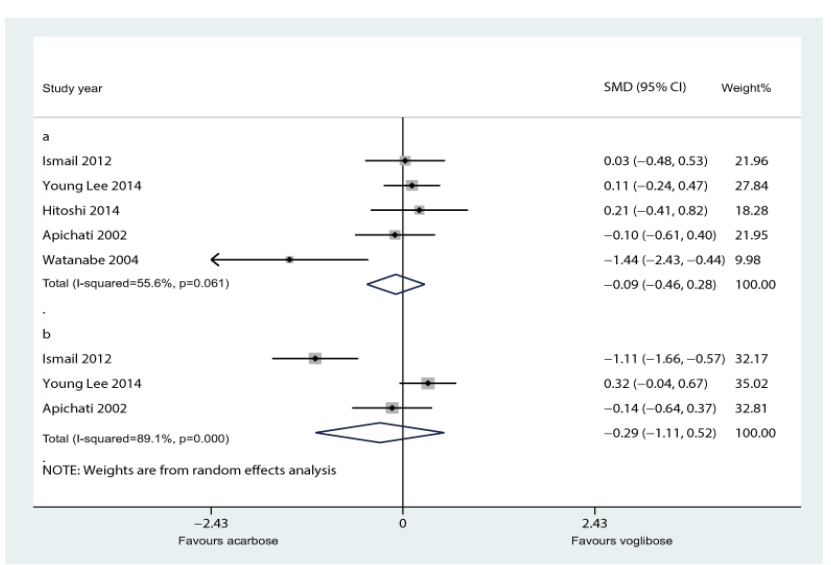

Figure 3: Standardised mean differences with $95 \%$ confidence interval in glycemic control parameters between acarbose group and voglibose group. a: FBG; b: Postprandial blood glucose.

\section{Outcomes}

$\mathbf{H b A}_{1 \mathrm{c}}$ : No significant difference was found on change of $\mathrm{HbA}_{1}$ between acarbose group and voglibose group (WMD 0.04; 95\% CI -0.39 to 0.47 ) (Figure 2).

FBG: No significant difference was found on change of FBG between acarbose group and voglibose group (SWM -0.09; 95\% CI -0.46 to 0.28 ) (Figure $3 a)$.

Postprandial blood glucose: No significant difference was found on change of postprandial blood glucose between acarbose group and voglibose group (SWM -0.29; 95\% CI -1.11 to 0.52) (Figure 3b).
Triglycerides: No significant difference was found on change of triglycerides between acarbose group and voglibose group (SWM -0.02 ; $95 \%$ CI -0.33 to 0.29 ) (Figure $4 a$ ).

Total cholesterol: No significant difference was found on change of total cholesterol between acarbose group and voglibose group (SWM 0.12 ; $95 \%$ CI -0.18 to 0.43 ) (Figure $4 \mathrm{~b}$ ).

LDL: No significant difference was found on change of LDL between acarbose group and voglibose group (SWM 0.15; 95\% CI - 0.15 to 0.46 ) (Figure 4c).

HDL: No significant difference was found on change of HDL between acarbose group and voglibose group (SWM 0.14; 95\% CI -0.17 to 0.45 ) (Figure $4 \mathrm{~d}$ ).

Fasting insulin: There was just one study [22] reported change of fasting insulin. Thus, it was impossible to compare.

Postprandial serum insulin: No significant difference was found on change of postprandial serum insulin between acarbose group and voglibose group (SWM 0.27; 95\% CI -0.12 to 0.66 ) (Figure $4 \mathrm{e}$ ).

Fasting glucagon: No any included studies reported change of fasting glucagon. Thus, no relevant data were analysed.

Postprandial serum glucagon: No any included studies reported change of postprandial serum glucagon. Thus, no relevant data were analysed.

Body weight: No significant difference was found on change of body weight between acarbose group and voglibose group (SWM 0.20; 95\% CI -0.85 to 0.45 ) (Figure 5).

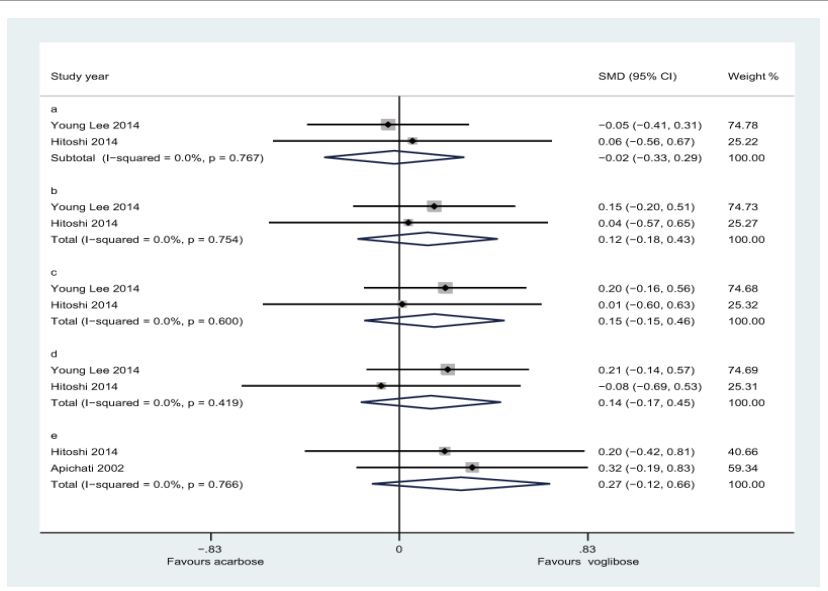

Figure 4: Standardised mean differences with 95\% confidence confidence interval in glycemic control parameters between acarbose group and voglibose group. a: Triglycerides; b: Total cholesterol; c: LDL; d: HDL; e: Postprandial serum insulin. 


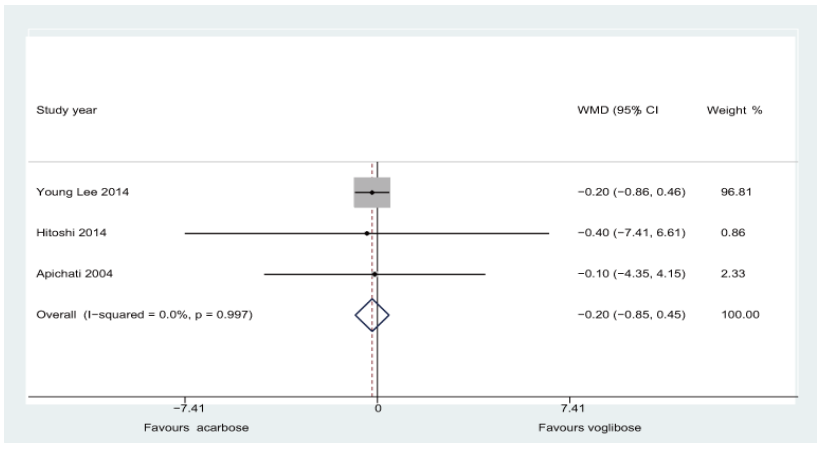

Figure 5: Weighted mean differences with 95\% confidence interval in body weight between acarbose group and voglibose group.

\begin{tabular}{|c|c|c|c|}
\hline Outcomes & Summery statistics & $\mathbf{9 5 \%} \mathbf{C l}$ & $\mathbf{I}^{\mathbf{2}}$ \\
\hline $\mathrm{HbA}_{1 \mathrm{c}}$ & WMD 0.04 & -0.39 to 0.47 & $77.8 \%$ \\
\hline FBG & SWM -0.09 & -0.46 to 0.28 & $55.6 \%$ \\
\hline Postprandial blood glucose & SWM -0.29 & -1.11 to 0.52 & $89.1 \%$ \\
\hline Triglycerides & SWM -0.02 & -0.33 to 0.29 & $0.0 \%$ \\
\hline Total cholesterol & SWM 0.12 & -0.18 to 0.43 & $0.0 \%$ \\
\hline LDL & SWM 0.15 & -0.15 to 0.46 & $0.0 \%$ \\
\hline HDL & SWM 0.14 & -0.17 to 0.45 & $0.0 \%$ \\
\hline Postprandial serum insulin & SWM 0.27 & -0.12 to 0.66 & $0.0 \%$ \\
\hline Body weight & SWM 0.20 & -0.85 to 0.45 & $0.0 \%$ \\
\hline
\end{tabular}

Notes: $\mathrm{HbA}_{1 \mathrm{c}}$ : Glycosylated Hemoglobin; FBG: Fasting Blood Glucose; LDL: Low Density Lipoprotein; HDL: High Density Lipoprotein; WMD: Weighted Mean Differences; SWM: Standardized Mean Differences; $\mathrm{Cl}$ : Confidence Interval

Table 3: Results of meta-analysis summary.

Hypoglycemia: There was just one study [11] reported hypoglycemia event. Thus, it was impossible to compare.

Hyperglycemia: No any included studies reported hyperglycemia event. Thus, no relevant data were analysed.

Adverse events: Most included studies [11,20-22] reported adverse events. However, these adverse events were various and there were no appropriate data to perform meta-analysis. Dr. Teli Shaikh Emaran Shaikh Ismail et al. [20] reported the following adverse events in acarbose group: nausea (1 patient), flatulence (6 patients), diarrhea (2 patients), abdominal pain (1 patient); in voglibose group, adverse event was flatulence ( 2 patients). Mi Young Lee et al. [11] reported that "a total of 137 adverse events in 44/60 (73.3\%) subjects in acarbose group and 143 adverse events in 42/62 (67.7\%) subjects in voglibose group", including gastrointestinal disorders, general disorders, metabolic disorders, eye disorders, nervous system disorders, respiratory disorders, skin disorders. Hitoshi Sugihara et al. [21] showed that the incidences of persistent borborygmus, abdominal distension and flatulence in acarbose group were higher than voglibose group. Apichati Vichayanrat et al. [22] showed that incidences of adverse events in acarbose group were significantly higher than voglibose group $(\mathrm{P}<0.05)$. From the above, adverse events in acarbose group were higher than voglibose group.

All results of meta-analysis were presented in Table 3.

Sensitivity analysis: For all pooled results, our result of sensitivity analysis showed that there was not a single study may influence the whole results.

Subgroup analysis: Our systematic review only included 5 studies and a small number of studies were difficult to be divided into a subgroup by race or dose. Thus, we did not perform subgroup analysis.
Meta-regression analysis: We could not find the sources of heterogeneity by meta- regression analysis.

Publication bias: Funnel plot always was used to exam publication bias for large sample size and it is difficult to exam publication bias for small sample size. Our systematic review only included 5 studies, so we did not exam publication bias.

\section{Discussion}

The goal of this systematic review was to compare the efficacy and safety of acarbose and voglibose for the treatment of patients with type 2 diabetes. We included in a total of five randomised trials and drew a conclusion: acarbose could not gain a better efficacy in blood sugar control and body weight compared with voglibose; in contrast, voglibose is safer than acarbose.

In terms of efficacy, there are no differences between acarbose and voglibose; in terms of safety, voglibose is safer than acarbose. Thus, between the choice of acarbose and voglibose should be recommended for the treatment of patients with type 2 diabetes. This recommendation has some limitations. Firstly, some included trials reported the shortterm efficacy and safety of acarbose and voglibose for the treatment of patients with type 2 diabetes. What is more, the patients with type 2 diabetes were different in age. Dosage of administration and administration route were also different. We did not perform subgroup analysis due to a small number of included trials. Significant heterogeneity was among the included trials, but we could not find it sources through sensitivity analysis and meta-regression. Instead, we selected random medol.

To our knowledge, a previous systematic review [14] had reported the effects of monotherapy with alpha-glucosidase inhibitors in patients with type 2 diabetes, but they only did a comparison of acarbose and miglitol versus placebo and sulfonylurea. The efficacy and safety of acarbose compared with voglibose in patients with type 2 diabetes have not been systematically summarized before. Our systematic review is the first one which systematically summarized the efficacy and safety of acarbose compared with voglibose in patients with type 2 diabetes.

Our systematic review had several limitations. We only included in five published studies, which was lack of credibility. In addition, we only included in English studies, and it was a limitation. There may be some unpublished studies which we cannot find, which caused a potential limitation. What is more, our included trials had methodological limitations. Three included trials [11,22-23] had unclear risk of bias in random sequence generation. All included trials [11,20-23] had unclear risk of bias in allocation concealment. One included trial [20] had high risk of bias in blinding of participants and personnel. Four included trials [11,21-23] had unclear risk of bias in blinding of participants and personnel. One trial [20] had high risk of bias in blinding of outcome assessment. Four included trials [11,21-23] had unclear risk of bias in blinding of outcome assessment. Finally, a limitation was caused due to lack of gray literatures (presentations, unpublished data, government reports, and other traditional or nontraditional sources of evidence).

\section{Conclusion}

Our insufficient evidence finds that voglibose is more suitable for the treatment of type 2 diabetes compared with acarbose. Further welldesigned and multicentric RCTs with a larger sample to evaluate the efficacy and safety of acarbose compared with voglibose in patients with type 2 diabetes are required. These trials will clearly address the efficacy and safety of acarbose compared with voglibose in patients with type 2 diabetes. 
Citation: Liu L, Su SW, Xu Y, Wan Q, Yang XL, et al. (2018) The Efficacy and Safety of Acarbose compared with Voglibose in Patients with Type 2 Diabetes: A Systematic Review and Meta-Analysis. J Mol Biomark Diagn 9: 375. doi: 10.4172/2155-9929.1000375

\section{Funding}

None

\section{Ethical Approval}

This article does not contain any studies with human participants or animals performed by any of the authors.

\section{References}

1. Krentz AJ, Bailey CJ (2005) Oral antidiabetic agents: Current role in type 2 diabetes mellitus. Drugs 65: 385-411.

2. Butler AE, Janson J, Bonner-Weir S, Ritzel R, Rizza RA, et al. (2003) $\beta$-cell deficit and increased $\beta$-cell apoptosis in humans with type 2 diabetes. Diabetes 52: $102-110$

3. Rydén L, Standl E, Bartnik M, Van den Berghe G, Betteridge J, et al. (2009) Guidelines on diabetes, pre-diabetes, and cardiovascular diseases. Euro Heart J 9: C3-C74.

4. Shaw JE, Sicree RA, Zimmet PZ (2010) Global estimates of the prevalence of diabetes for 2010 and 2030. Diabetes res cln pract 87: 4-14.

5. Schmidt DD, Frommer W, Junge B, Muller L, Wingender W, et al. (1977) alphaGlucosidase inhibitors. New complex oligosaccharides of microbial origin. Die Naturwissenschaften 64: 535-536.

6. Caspary WF, Graf S (1979) Inhibition of human intestinal alphaglucosidehydrolases by a new complex oligosaccharide. Res Exp Med (Berl) 175: $1-6$

7. Puls W, Keup U, Krause HP, Thomas G, Hoffmeister F, et al. (1977) Glucosidase inhibition. A new approach to the treatment of diabetes, obesity, and hyperlipoproteinaemia. Die Naturwissenschaften 64(10): 536-537.

8. Coniff R, Krol A (1997) Acarbose: A review of US clinical experience. Clin Ther 19: 16-26.

9. Scheen AJ (1997) Drug treatment of non-insulin-dependent diabetes mellitus in the 1990s. Achievements and future developments. Drugs 54: 355-368.

10. Fujisawa T, Ikegami H, Inoue K, Kawabata Y, Ogihara T (2005) Effect of two alpha-glucosidase inhibitors, voglibose and acarbose, on postprandial hyperglycemia correlates with subjective abdominal symptoms. Metabolism 54:387-390.

11. Lee MY, Choi DS, Lee MK, Lee HW, Park TS, et al. (2014) Comparison of acarbose and voglibose in diabetes patients who are inadequately controlled with basal insulin treatment: Randomized, parallel, open-label, active-controlled study. J Korean Med Sci 29: 90-97.
12. Van De Laar FA, Lucassen PLBJ, Akkermans RP, Van De Lisdonk $E H$, De Grauw WJC (2006) Alpha-glucosidase inhibitors for people with impaired glucose tolerance or impaired fasting blood glucose. Cochrane Database Syst Rev 2006: CD005061.

13. Cai X, Yang W, Zhou L, Zhang S, Han X, et al. (2015) Comparisons of the efficacy of glucose control, lipid profile, and $\beta$-cell function between DPP4 inhibitors and $\mathrm{AGI}$ treatment in type 2 diabetes patients: a meta-analysis. Endocrine 50: 590-597.

14. Van de Laar FA, Lucassen PL, Akkermans RP, Van de Lisdonk EH, Rutten $\mathrm{GE}$, et al. (2005) Alpha-glucosidase inhibitors for patients with type 2 diabetes: results from a Cochrane systematic review and meta-analysis. Diabetes care 28: $154-163$.

15. Moher D, Liberati A, Tetzlaff J, Altman DG (2009) Preferred reporting items for systematic reviews and meta-analyses: the PRISMA statement. BMJ 339 : b2535.

16. Higgins JP, Altman DG, Gotzsche PC, Juni P, Moher D, et al. (2011) The cochrane collaboration's tool for assessing risk of bias in randomised trials. BMJ 343:d5928.

17. Higgins JP, Thompson SG (2002) Quantifying heterogeneity in a meta-analysis Stat Med 21: 1539-1558

18. DerSimonian R, Laird N (1986) Meta-analysis in clinical trials. Control Clin Trials 7: 177-188.

19. Mantel N, Haenszel W (1959) Statistical aspects of the analysis of data from retrospective studies of disease. J Natl Cancer Inst 22: 719-748.

20. Ismail TSES, Deshmukh SA (2012) Comparative study of effect of alpha glucosidase inhibitors-miglitol, acarbose and voglibose on postprandia hyperglycemia and glycosylated hemoglobin in type-2 diabetes mellitus. International Journal of Pharma and Bio Sciences 3: 337-343

21. Sugihara $H$, Nagao $M$, Harada $T$, Nakajima $Y$, Tanimura-Inagaki $K$, et al (2014) Comparison of three alpha-glucosidase inhibitors for glycemic contro and bodyweight reduction in Japanese patients with obese type 2 diabetes. $J$ Diabetes Investig 5: 206-212

22. Vichayanrat A, Ploybutr S, Tunlakit M, Watanakejorn $P$ (2002) Efficacy and safety of voglibose in comparison with acarbose in type 2 diabetic patients. Diabetes Res Clin Pract 55: 99-103.

23. Watanabe K, Uchino H, Ohmura C, Tanaka Y, Onuma T, et al. (2014) Different effects of two alpha-glucosidase inhibitors, acarbose and voglibose, on serum 1,5-anhydroglucitol (1,5AG) level. J Diabetes Complications 18: 183-186. 